“C 2019 IEEE. Personal use of this material is permitted. Permission from IEEE must be obtained for all other uses, in any current or future media, including reprinting/republishing this material for advertising or promotional purposes, creating new collective works, for resale or redistribution to servers or lists, or reuse of any copyrighted component of this work in other works." 


\title{
Design of Planar, Wideband, Multi-Polarization Reconfigurable Filtering Antenna
}

\author{
Dajiang $\mathrm{Li}^{1}$, Ming-Chun Tang ${ }^{1}$, Xiaoming Chen ${ }^{1}$, Yang Wang ${ }^{1}$, Kun-Zhi Hu ${ }^{1}$, and Richard W. Ziolkowski ${ }^{2}$ \\ ${ }^{1}$ School of Microelectronics and Communication Engineering, Chongqing University, Chongqing, China \\ tangmingchun@cqu.edu.cn \\ ${ }^{2}$ Global Big Data Technologies Centre, University of Technology Sydney, Ultimo NSW 2007, Australia \\ Richard.Ziolkowski@uts.adu.au
}

\begin{abstract}
A planar, wideband, multi-polarization reconfigurable filtering antenna is proposed. By controlling the state of PIN diodes, the operation states of the filtering antenna can be switched among linear polarization (LP), left-hand circular polarization (LHCP) and right-hand circular polarization (RHCP). The filtering antenna consists of a driven patch, a parasitic patch, and an established reconfigurable feed network. Two radiation nulls can be generated at the lower and upper band-edge in all operation states through interaction between the driven patch and feeding network. A pair of U-slots is etched on the driven patch to obtain the extra radiation null to further improve the sharp roll-off rate at the upper band-edge. The optimized prototype was fabricated and tested. The measured results, in good agreement with the simulated values, demonstrate that the filtenna has a wide $-10 \mathrm{~dB}$ fractional bandwidth: $15.6 \%$, a realized gain value over operational bandwidth of $7.7 \pm 0.5 \mathrm{dBi}$, and stable radiation performance. Furthermore, the filtenna presents a good out-of-band rejection level.
\end{abstract}

Index Terms - wideband, multi-polarization, reconfigurable, radiation nulls, filtering antenna.

\section{INTRODUCTION}

Increasingly congested spectrum resources and complex electromagnetic environments have led to tremendous expectations for antennas with reconfigurable characteristics [1] and multi-functions [2]. As a result, polarization reconfigurable antennas [3-4] have become an attractive choice with their attributes of increasing the system capacity and enhancing an anti-interference ability. The same is true of filtering antennas [5-6] with their inviting features of additional frequency selectivity and out-of-band rejection to effectively improve the signal-to-noise ratio.

Polarization reconfigurable filtering antennas (PRFAs) [7-9] integrate both the polarization reconfigurability and the filtering capability into a single module. The hope is to achieve the best of both systems, i.e., increasing the anti-interference performance and improving the channel capacity while reducing the RF system costs. However, relatively few researches about PRFAs have been made. A non-planar waveguide structure was designed in [7], but it has a high profile. On the other hand, planar structures were adopted in [8] and [9]. Nevertheless, they have narrow bandwidths.

In this paper, a planar, wideband, multi-polarization reconfigurable filtering antenna is reported. In contrast to the reconfigurable two CP-state systems reported in [9], it possesses three switchable polarization states, i.e., LP, LHCP, and
RHCP states. Moreover, it has a more compact size: $0.48 \lambda_{0} \times$ $0.45 \lambda_{0}$, low profile: $0.096 \lambda_{0}$, where $\lambda_{0}$ is the free space wavelength corresponding to the center frequency of the operational band, and a much wider operational bandwidth, $15.6 \%$, than the ones disclosed in [8] and [9].

\section{DESIGN OF THE PRFA}

Fig. 1 illustrates the PRFA design, which consists of three square substrate layers labeled as Layer_1, Layer_2 and Layer_3, respectively. All of the three substrates are Rogers $\mathrm{RT} /$ Duroid 5880 with a permittivity of $\varepsilon_{r}=2.2$, a thickness of $0.787 \mathrm{~mm}$, and a size of $L_{l} \times W_{l}=120 \times 120 \mathrm{~mm}^{2}$. An air gap is introduced between Layer_1 and Layer_2 to enhance the antenna bandwidth and gain. With reference to Fig. 1(a), a square parasitic patch is fabricated on the upper surface of Layer_1. The driven patch, which is etched with a pair of Uslots to introduce an additional radiation null at the upper stopband, is fabricated on the upper surface of Layer_ 2 .

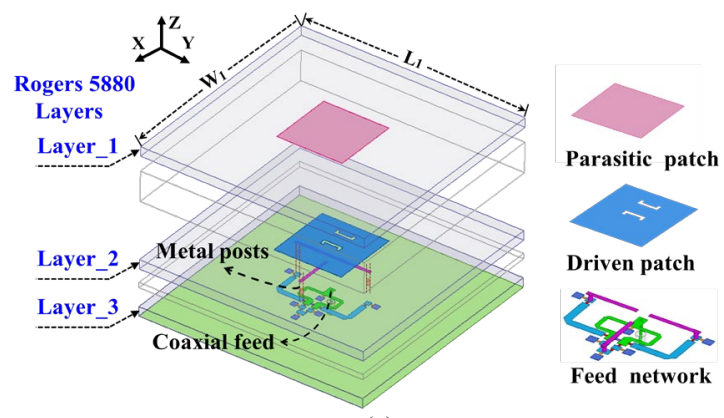

(a)

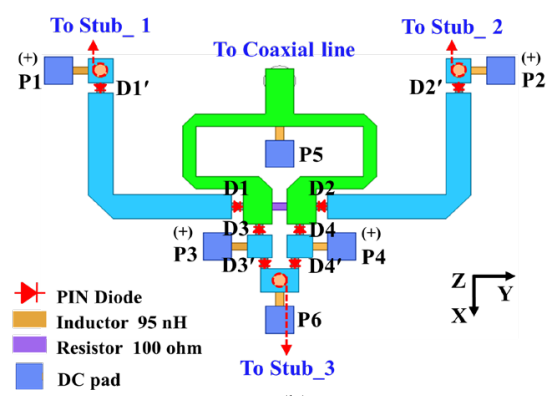

(b)

Fig.1 Geometry of the proposed PRFA. (a) 3-D isometric view. (b) The upper surface of layer 3 . 
A reconfigurable feed network was developed to realize the polarization reconfigurability amongst the one LP and two CP states. It also introduces two radiation nulls in the lower stopband and upper stop-band to obtain the filtering capability, respectively. It is composed of a Wilkinson power divider, a set of phase-shift strips, and three coupling stubs. The Wilkinson power divider (green) and the phase-shift strips (blue) are all mounted on the upper surface of Layer 3. The three coupling stubs (magenta), labeled as Stub_1, Stub_2 and Stub_3 with the same size, are located on the lower surface of Layer_2. The coupling stubs and the phase-shift strips are connected by three metal posts (yellow).

As shown in Fig. 1(b), eight Infineon PIN diodes (D1\&D1', D2\&D2', D3\&D3', D4\&D4') in SC79 packages and six $95 \mathrm{nH}$ inductors in 0603 packages are integrated into the lower part of feed network. Each diode can be forward biased to ON state with a dc voltage $(0.95 \mathrm{~V})$, while it will be in OFF state if left unbiased. To provide de voltage (P1-P6) to the diodes, six square dc pads are built next to the feedline, and connected to it via six inductors with the function of blocking any RF signal from entering into the dc bias network.

\section{SimULATED AND MEASURED RESUltS}

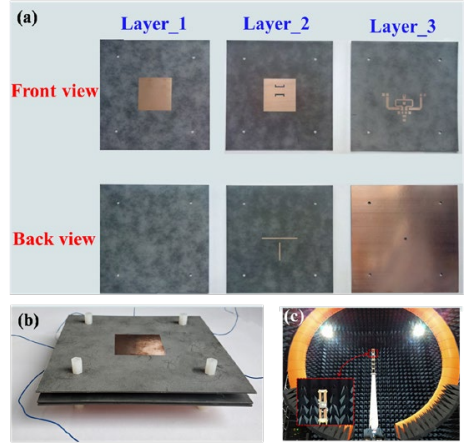

Fig. 2. Fabricated prototype of the proposed PFRA. (a) Front and back views of each layer before assembly. (b) 3-D view of the assembled antenna mounted. (c) The antenna under test in the anechoic measurement chamber.

As is shown in Fig. 2, the reconfigurable filtenna was fabricated, assembled, and tested. The measured results in Fig. 3 are in good agreement with their simulated values. They indicate that the filtenna exhibits a wide $15.6 \%$ effective fractional bandwidth (FBW) centered at $2.9 \mathrm{GHz}$ with a flat broadside average realized gain, $7.7 \pm 0.5 \mathrm{dBi}$. The corresponding simulated radiation efficiency (RE) values are all over $75 \%$ within this operational band. Note that the effective bandwidth refers to the overlap of the bandwidths of all three states. Moreover, three radiation nulls (one in the lower stop-band and two in the upper stop-band) were produced. As a consequence, the filtenna exhibits a good out-of-band rejection performance in all of its three polarization states. The simulated and measured results of FBW and realized gain in all three states, and axial ratio (AR) FBW in both CP states, are summarized in Table I for easy reference.

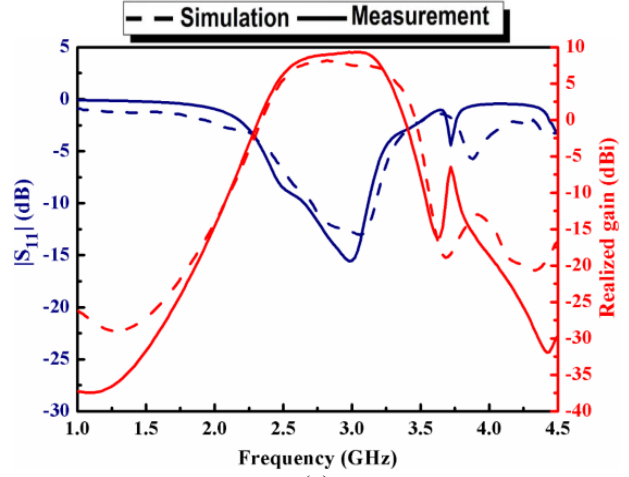

(a)

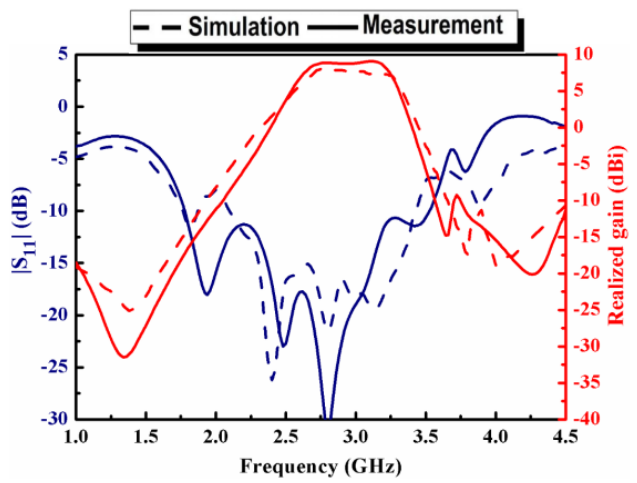

(b)

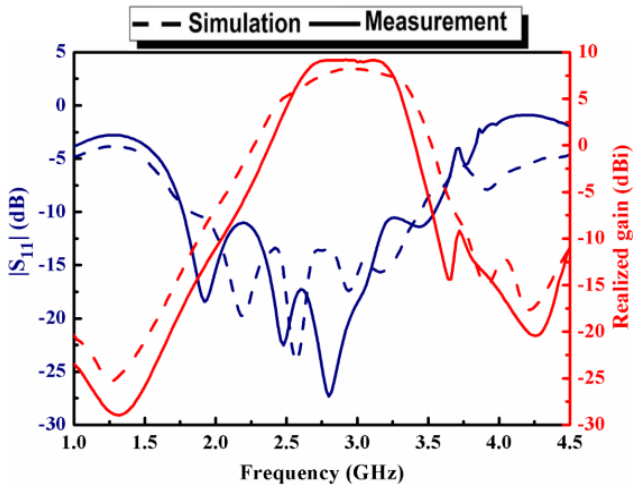

(c)

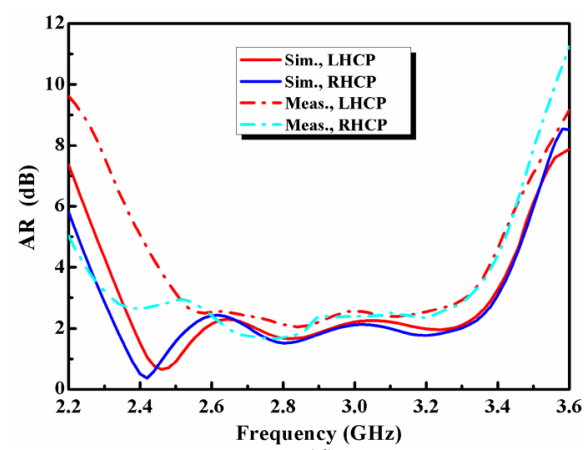

(d)

Fig. 3 Simulated and measured $\left|S_{11}\right|$, realized gains, and AR values of the proposed PRFA. (a) The LP states. (b) The LHCP state. (c) The RHCP state. (d) AR values of the LHCP and RHCP states. 
TABLE I

Simulated and MEASURed Results for The PRFA in All of Its PolariZATION StATES. THE MEASURED (SiMUlATED) RESUlts ARE IN THE SHADED (CLEAR) BOXES

\begin{tabular}{|c|c|c|c|}
\hline State & LP & LHCP & RHCP \\
\hline $\begin{array}{c}\text { Impedance FBW (\%) } \\
\left(\left|\mathrm{S}_{11}\right| \leq-10 \mathrm{~dB}\right)\end{array}$ & 16.3 & 66.4 & 67 \\
\cline { 2 - 4 } & 15.6 & 62.6 & 62.6 \\
\hline $\begin{array}{c}\text { Average realized gain } \\
(\mathrm{dBi})\end{array}$ & 8.8 & 8.6 & 8.6 \\
\cline { 2 - 4 } & 7.7 & 7.7 & 7.7 \\
\hline $\begin{array}{c}\text { AR FBW (\%) } \\
(\mathrm{AR} \leq 3 \mathrm{~dB})\end{array}$ & & 34.5 & 38.7 \\
\cline { 2 - 4 } & & 27.5 & 34.9 \\
\hline
\end{tabular}

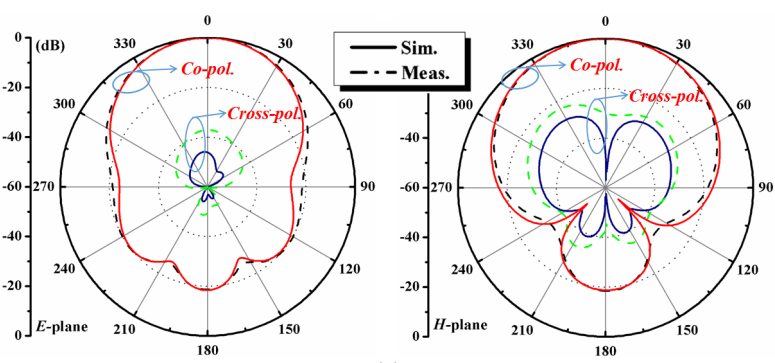

(a)



(b)

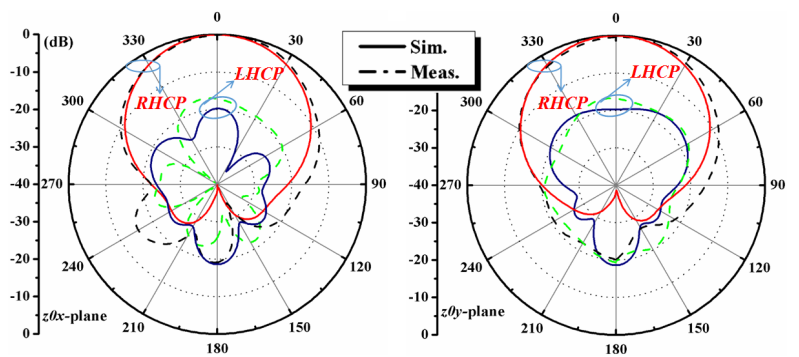

(c)

Fig. 4 The simulated and measured normalized radiation patterns of the PRFA operating at $2.9 \mathrm{GHz}$ (the center frequency point of the effective overlapped BW) for all three states. (a) LP state. (b) LHCP state. (c) RHCP state.

Fig. 4 presents the simulated and measured realized gain patterns for the three polarization states at $2.9 \mathrm{GHz}$. The mainbeam direction for each polarization state is clearly broadside and the patterns are symmetrical about this direction. The feature arises from the symmetric feed network and patch structure. The measured co-polarized fields in its LP state are at least $37 \mathrm{~dB}$ stronger than the cross-polarized counterparts. The measured LHCP (RHCP) fields are at least $17 \mathrm{~dB}$ stronger than the opposite state. Additionally, the measured front-to- back ratio is greater than $18 \mathrm{~dB}$ for all three polarization states.

\section{CONCLUSION}

A planar, wideband, multi-polarization reconfigurable filtering antenna was demonstrated. A set of PIN diodes integrated into the feed network allows one to dynamically reconfigure its three polarization states (LP, LHCP, and RHCP). A prototype was fabricated, and tested. The measured results, in good agreement with their simulated values, demonstrate that the antenna exhibits a wide $15.6 \% \mathrm{FBW}$ centered at $2.9 \mathrm{GHz}$ with a flat broadside average realized gain, $7.7 \pm 0.5 \mathrm{dBi}$, and corresponding simulated $\mathrm{RE}$ values over $75 \%$ over this operational band. Moreover, the measured gain responses with three radiation nulls for all three states demonstrate good out-ofband rejection performance. The advantageous design and performance characteristics make the filtenna very suitable for practical applications in wireless communication systems, especially in complex electromagnetic environments.

\section{REFERENCES}

[1] M.-C. Tang, B. Zhou, and R. W. Ziolkowski, "Low-profile, electrically small, Huygens source antenna with pattern-reconfigurability that covers the entire azimuthal plane," IEEE Trans. Antennas Propag., vol.65, pp. 1063-1072, March 2017.

[2] P. R. Herczfeld, A. S. Daryoush, A. Rosen, A. K. Sharma, and V. M. Contarino, "A Cross-Shaped Dielectric Resonator Antenna for Multifunction and Polarization Diversity Applications," IEEE Antennas Wireless Propag. Lett., vol.10, pp. 742-745, 2011.

[3] M.-C. Tang, Z. Wu, T. Shi, and R. W. Ziolkowski, "Electrically small, low-profile, planar, Huygens dipole antenna with quad-polarization diversity," IEEE Trans. Antennas Propag., vol. 66, no. 12, pp. 6772-6780, Dec. 2018

[4] F. Wu and K. M. Luk, "Single-port reconfigurable magneto-electric dipole antenna with quad-polarization diversity," IEEE Trans. Antennas Propag., vol. 65, no. 5, pp. 2289-2296, May 2017.

[5] M.-C. Tang, Y. Chen, and R. W. Ziolkowski, "Experimentally validated, planar, wideband, electrically small, monopole filtennas based on capacitively loaded loop resonators," IEEE Trans. Antennas Propag., vol. 64, no. 8, pp. 3353-3360, Aug. 2016.

[6] K.-Z. Hu, M.-C. Tang, M. Li, and R. W. Ziolkowski, "Compact, lowprofile, bandwidth-enhanced substrate integrated waveguide filtenna," IEEE Antennas Wireless Propag. Lett., vol. 17, pp. 1552-1556, 2018.

[7] F. Farzami, S. Khaledian, B. Smida, and D. Erricolo, "Reconfigurable linear/circular polarization rectangular waveguide filtenna," IEEE Trans. Antennas Propag., vol. 66, no. 1, pp. 9-15, Jan. 2018.

[8] T. H. Gan, Z. Yang, E. L. Tan, J. X. Lim, M. Q. Huynh, and M. Mayank, "A polarization-reconfigurable filtering antenna system," IEEE Antennas Propag. Magazine., vol. 55, no. 6, pp. 198-219, Dec. 2013.

[9] Y. Lu, Y. Wang, S. Gao, C. Hua, and T. Liu, "Circularly polarised integrated filtering antenna with polarisation reconfigurability," IET Microw. Antennas Propag., vol. 11, pp. 2247-2252, Dec. 2017. 